\title{
Revisiting anatomical landmarks of cadaveric nerve-sparing radical prostatectomy in accordance to Terminologia Anatomica
}

\author{
Emre Huri $^{1,2}$, İlkan Tatar ${ }^{2}$, Mustafa Fevzi Sargon ${ }^{2}$, Cankon Germiyanoğlu ${ }^{1}$, Ruhgün Başar ${ }^{2}$ \\ ${ }^{I}$ Second Urology Clinic, Ankara Training and Research Hospital, Ankara, Turkey \\ ${ }^{2}$ Department of Anatomy, Faculty of Medicine, Hacettepe University, Ankara, Turkey
}

\begin{abstract}
Objectives: Because of the developing and standardizing urology resident education, we aimed to show important anatomical landmarks of nerve-sparing radical prostatectomy in a cadaveric dissection.

Methods: The steps of the dissection were; 1) Incision of pelvic fascia, 2) Dissection of the puboprostatic complex, 3) Dissection of the urethral sphincteric complex and apical dissection of the prostate and urethra, 4) Dissection of the posterior part of the prostate, 5) Preservation of the neurovascular bundle(included the cavernous nerve of the penis), pedicle dissection, and 6) Dissection of bladder neck, seminal vesicle and ductus deference.

Results: At the end of the dissection, proved anatomical landmarks were; 1) parietal pelvic fascia, tendinous arc, internal obturator fascia, 2) puboprostatic ligament, pubic symphysis, deep dorsal penile vein, 3) external urethral sphincter, levator ani muscle, neurovascular bundle, 4) rectoprostatic fascia, seminal vesicles, perirectal fat tissue, 5) cavernous nerve, capsular artery and vein, levator fascia, periprostatic fascia, prostatic capsule, 6) Bladder neck, deferent duct, detrusor apron.

Conclusion: Uniformity of terminology and identical definitions of structures in both cadaveric dissection and surgery might help to improve comprehensibility of anatomical and surgical knowledge of the young urological surgeons and anatomists.
\end{abstract}

Key words: cadaver; radical prostatectomy; Terminologia Anatomica

Anatomy 2011;5:18-22, (C) 2011 TSACA

\section{Introduction}

Currently, nerve-sparing radical prostatectomy (nSRP), whether laparoscopic or open technique, is a well-established surgical procedure for the treatment of localized prostate cancer. Technical details of this procedure make the operation more difficult to performed by surgeons. Indeed, Stolzenburg et al. determined the anatomical landmarks of radical prostatectomy (RP) ${ }^{[1]}$ However, in both techniques, the surgical and functional outcomes are comparable. ${ }^{[2]}$ Advances in anatomical elucidation of the prostate and in surgical techniques have contributed to excellent survival and functional results after RP. ${ }^{[3-8]}$ Preservation of important functional anatomic landmarks during the dissection in RP is closely related with significant functional outcomes in the postoperative period.
Despite the different approaches for RP (open or endoscopic), the key point for better results is to be aware of anatomical understanding. ${ }^{[1]}$ There are various studies advocating the anatomy and nomenclature of the structures of this area of interest. The importance of using Terminologia Anatomica, ${ }^{[0]}$ which was updated in 1998, should also be stressed for showing anatomic landmarks in RP. The cadaver model was used to determine detailed anatomical structures during radical prostatectomy. This study will give a detailed description of the prostatic surgical anatomy in the light of cadaveric images, showing details usually not easily recognized while performing live surgery. However, we believe that the collaboration between the urologists and anatomist will improve the clarification of exact anatomic struc- 
tures and landmarks during radical prostatectomy, which still has controversial anatomic points.

In this study, we evaluated the efficacy of cadaveric $\mathrm{nSRP}$ as a novel surgical training model in accordance with anatomic landmarks.

\section{Materials and Methods}

The theoretical model of nSRP was based on this study. Retropubic nSRP was performed on a male cadaver by urologist (E.H.) at Hacettepe University, Laboratory of Anatomy Department. The operation was supervised by anatomists (M.F.S. and İ.T.). The important anatomic landmarks in nSRP were pointed out in the cadaver model by urologists and anatomists. The procedure was divided into six steps according to the anatomic relations. In this classification, Terminologia Anatomica and identification of functional anatomy were included. Steps were determined as; 1) Incision of parietal pelvic fascia (endopelvic fascia): the initial step to reach the lateral prostate surface, 2) Detachment of puboprostatic complex, including ligaments and dorsal venous complex: one of the basic structures for preserving the unexpected bleeding, 3) Urethral sphincteric units and anatomical apical dissection of prostate and urethra, 4) Dissection of rectoprostatic fascia (Denonvilliers' fascia), posterior part of the prostate, seminal vesicle and ductus deferens, 5) Preservation of lateral neurovascular bundle and pedicle of prostate dissection, the relation with pelvic plexus, and 6) Accurate bladder neck dissection, detrusor apron and ductus deferens.

The anatomical landmarks for each surgical step were determined. However, the functional importance of anatomic structures was stressed.

\section{Results}

The operation lasted approximately 1.5 hours. Each step of the procedure was carried out carefully in accordance with anatomic rules. The anatomic landmarks were identified according to the Terminologia Anatomica. Incision was performed onto the anterior abdominal wall from inferior umbilicus to the symphisis pubis along the linea alba. The important anatomical structures are linea alba, arcuate line, semilunar line, umbilicus, rectus abdominis muscle, superior and inferior epigastric artery, and veins. In the first step, the lateral vertical incision was performed onto the parietal pelvic fascia (endopelvic fascia) (Figure 1). Tendinous arc was determined as a landmark for proper incision. The incision was completed at the lateral side of puboprostatic ligament. The other anatom- ic landmarks are internal obturator fascia and iliac fascia, branches of internal pudental artery. Both fascias were seen as a part of the parietal pelvic fascia. Possible pitfalls are bleeding from the branches of internal pudental artery, fibers of levator ani muscle, uncorrected plane at the lateral wall of prostate and undesirable view at the prostatovesical junction. In the second step, the puboprostatic detachment of puboprostatic complex was dissected to reach the apical side of the prostate. In this step, the anatomic landmarks were puboprostatic ligament, dorsal venous complex (Santorini plexus), symphysis pubis (pubic symphysis), superficial and deep dorsal penile vein, prostatic and vesical venous plexus. Possible pitfalls were undesirable bleeding from the Santorini plexus and superficial dorsal vein, inadequate plane through the apical prostate, injury to the neurovascular bundle at both side of the urethra. In the third step, the urethral sphincteric unit was identified. The external sphincter ascertains continence after RP. Anatomic landmarks are briefly external urethral sphincter muscle and levator ani muscle (pelvic floor support). Apical dissection of the prostate and membranous urethra without injuring the external sphincter unit yere performed. The most important landmarks are dorsal venous complex at the apical region, levator ani muscle, neurovascular bundle at the lateral side of urethra and urethral smooth muscle. Possible pitfalls were damage to the urethral sphincteric complex that could be a reason of incontinence, positive apical surgical margin and inadequate urethral length for vesicourethral anastomosis. In the fourth part, dissection of the posterior division of the prostate, the seminal vesicles and ductus deferenses were shown (Figure 2). The critical anatomic structures are rectoprostatic fascia (Denonvilliers' fascia), posterior part of prostate, seminal vesicles, perirectal fat tissue and ductus deferenses. Possible pitfalls are injury to rectum, seminal vesicles and pelvic plexus at the tip of the seminal vesicles. In the fifth part of the operation, dissection of lateral neurovascular structures and intrafascial or interfascial dissections were shown (Figure 3). Inferior vesical artery and branches, cavernous nerve of the penis, capsular artery and vein, visceral pelvic fascia, levator fascia (interfascial dissection), periprostatic fascia (intrafascial dissection), prostatic capsule, pelvic plexus and hypogastric nerve are important anatomic landmarks. Possible pitfalls are bleeding from inferior vesical artery branches named as the prostatic pedicle, injury to the predominant neurovascular bundle and damage to the nerves surrounding the bladder neck. In the last part, the proper bladder neck dissection was performed (Figure 4). Detrusor apron is a landmark point for starting the anterior dissection of 


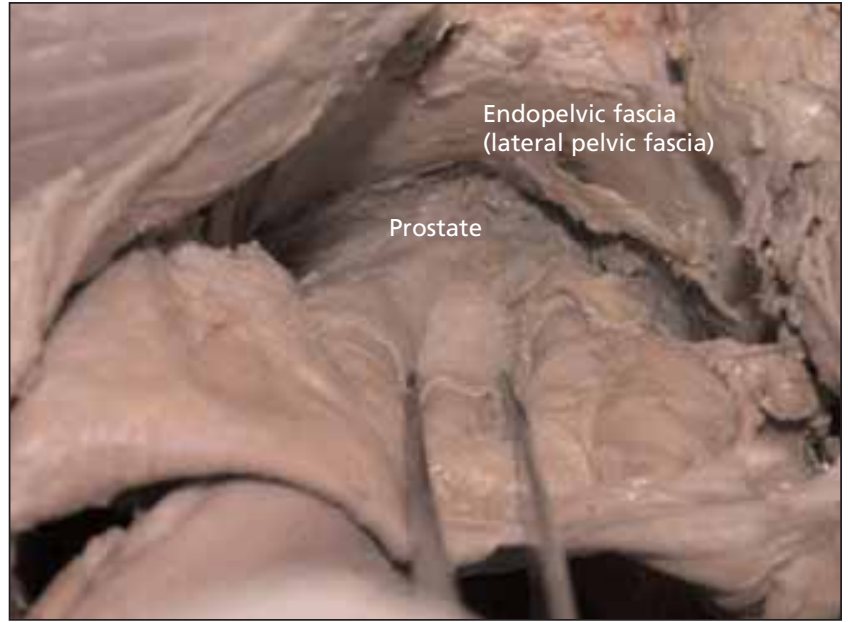

Figure 1. The cadaveric view of incised endopelvic fascia.



Figure 3. Intrafascial and interfascial dissection on the lateral prostate surface.

bladder neck. Posterior dissection of bladder neck should be performed far from ureteral orifices near to prostate base. Rectoprostatic fascia was seen posterior incision of bladder neck. Ductus deferens was seen at the medial, seminal vesicles were seen at the lateral side.

\section{Discussion}

Recent publications described the most important anatomical landmarks in the steps of nSRP. ${ }^{[1,10]}$ Understanding of the anatomic structure of each step of nSRP is of great interest due to the need to perform surgery accurately, to preserve the nerves necessary for erection and to provide full continent patient with protection of urinary sphincters. Additionally, efforts were

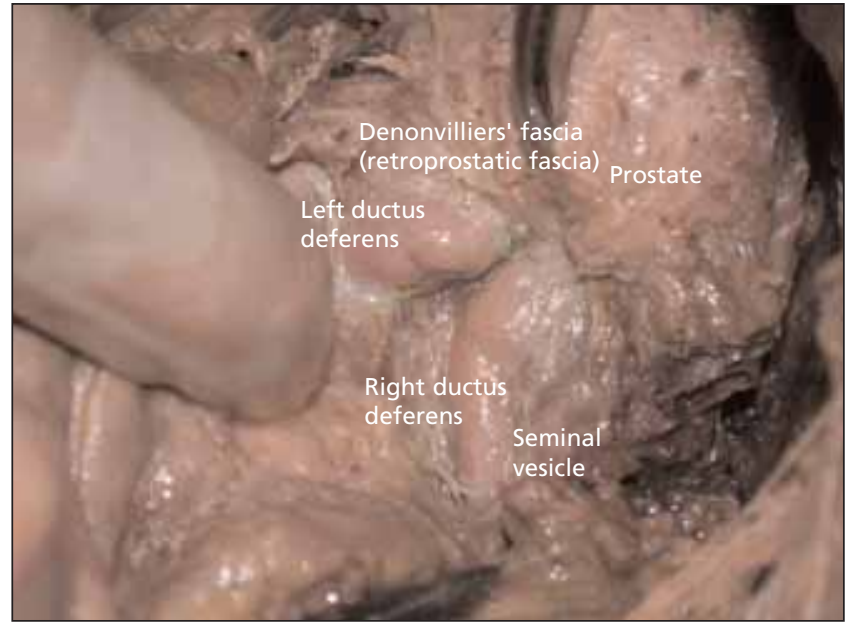

Figure 2. The cadaveric view of posterior part of prostate, seminal vesicle and ductus deference.

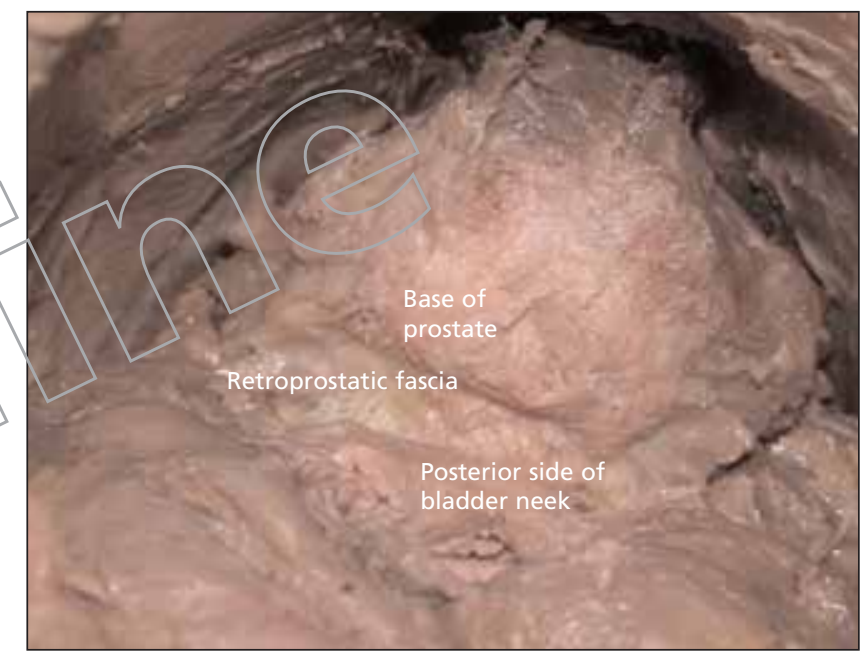

Figure 4. Bladder neck dissection (anterior and posterior parts).

being made to unify the existing terminology of the anatomic structures surrounding the prostate and related vessels, nerves and fascial parts. ${ }^{[11-15]}$ In Turkey, the general practical use of the official anatomical terminology is preferred in Latin. However, we believe that the similarities between clinical and official anatomical terminology should be provided in Latin and English. The misusage of the clinical Latin/English equivalents for some liver terms in Terminologia Anatomica was determined by Matusz. ${ }^{[16]}$ In this study, the correct clinical terminology was determined by combined support of urologists and anatomists. The standardization of clinical and official anatomic terminology will provide precise clinical advantage for identifying the anatomical landmarks. 
Myers et al. ${ }^{[17]}$ provided a new table of terms for the prostate with recommended amendments and deletions to existing official nomenclature as contained in the 1998 Terminologia Anatomica.

Stolzenburg et al. ${ }^{[2]}$ stressed the correct trocar placement for intrafascial nerve-sparing extraperitoneal radical prostatectomy with attention to the course of epigastric vessels. Although we performed open RP, we tried to describe the anatomical landmarks for trocar placement avoiding damage to the epigastric vessels.

The pelvic fascia covers the pelvic organs. This fascia is referred to as the endopelvic fascia (EPF) by some authors. ${ }^{[18,19]}$ It has two compartments, which are parietal and visceral. The surgeon gains full access to the prostate only after incision of the endopelvic fascia at the fusion between the parietal and visceral component of the antero-lateral corner of the prostate. ${ }^{[20-22]}$ The importance of the endopelvic fascia incision is not only to reach the prostate but also to determine the correct anatomic point for intrafascial dissection, although many surgeons today no longer incise the EPF during intrafascial dissection. The related structures with endopelvic fascia (parietal pelvic fascia) are the fascia of the levator ani muscle and lateral pelvic fascia, which is located at the ventral and lateral surface of the prostate under the $\mathrm{EPF}$ and extends in a posterior direction to cover also the neurovascular bundle (NVB) and the rectum. ${ }^{[14,8,8,2]}$ $\mathrm{EPF}$ is attached to the pubic bone and forms the puboprostatic ligaments (PPLs). The incision of EPF during intrafascial dissection takes place only ventrally and medially to the PPLs. ${ }^{[2]}$ However, PPLs support the external striated urethral sphincter and maintain the urethra in its normal location in the pelvic floor ${ }^{[1]}$ for earlier return of continence with bladder neck preservation technique. However, equivalent final outcomes were detected with puboprostatic ligament-sparing or a combination of both. ${ }^{[23]}$ Myers described the PPLs as pubovesical ligaments and showed that PPLs insert at the prostate base. ${ }^{[2]}$ We confirmed the proper structures of $\mathrm{EPF}$, lateral pelvic fascia, prostatic capsule, puboprostatic ligament and prostatic apex in cadaver.

Myers mentioned the importance of the detrusor apron, which partly obscures the prostate. ${ }^{[24]}$ Thus, the meticulous dissection of the antero-lateral aspect of the prostate and PPLs are crucial points in order to create an appropriate surgical plan for preservation of NVB. We detected detrusor apron and started the bladder neck dissection. PPLs have close anatomic relations with the superficial and deep dorsal penile veins and branches, which are termed the Santorini plexus. Avoidance of bleeding while dissecting the venous plexus should be the main target for an optimal anatomic view. In general, the branches of the deep dorsal penile vein spread to the anterior and lateral surfaces of the prostate at the cranial site. Therefore, the dissection of the dorsal venous complex is generally recommended near to the symphysis pubis. Santorini plexus was demonstrated in cadaver following the extraction of prostate.

Apical dissection includes dissection of the anterior urethra which is performed from lateral to medial and in three steps; dissection of Santorini plexus, dissection of prostatic apex and urethra with external sphincter muscle and dissection of the inner smooth muscle layer of the urethra. ${ }^{[2]}$ During RP, injury to the nerves of the intrapelvic branches and perineal branches of the pudendal nerve may occur during apical dissection of the prostate. Therefore, a meticulous dissection of the apical prostate is critically important in order to preserve urinary continence. ${ }^{[25]}$ The sphincter is separated from the dorsal venous complex (DVC) by its own muscle fascia. This fascia can be used as a landmark to limit dissection during this stage of RP and to ensure that the sphincter muscle remains intact. ${ }^{26}$ The urethral sphincter consists of two different muscle types. The outer muscle layer which is horseshoe-shaped and consists of striated muscle fibers, while the inner muscle layer surrounds the urethra completely and consists of smooth muscle fibers. ${ }^{[19]}$ During the dissection, avoidance of injuries to the NVB is mandatory. The urethra is separated from the NVB by the fascia of the external sphinteric muscle on the postero-lateral aspect. ${ }^{[26]}$ We could not separate DVC from the external urethral sphincter and urethra in cadaver, so this is our restrictive factor. The reason could be the fixation method of cadaver.

The inferior hypogastric plexus (IHP), which contains fibers from the both sympathetic and parasympathetic systems, is responsible for the mechanisms of erection, ejaculation and urinary continence. ${ }^{[27]}$ However, the main part of the IHP gives rise to the cavernous nerve $(\mathrm{CN})$, which is responsible for the erectile function. ${ }^{[2]}$ $\mathrm{CN}$ and IHP generally run in a caudal direction. They are often accompanied by vascular structures, which is the reason why this complex is often referred to as the NVB ${ }^{[12]}$ It consists not only of nerve fibers of the $\mathrm{CN}$ but also of nerve fibers designated for the innervations of the structures such as the urethral sphincter. Vascular structures are divided into two groups; arteries and veins. There are three arterial zones within the prostatic parenchyma: anterior or capsular, intermediate, and urethral. The blood supply of the prostate is derived prima- 
rily from the inferior vesicle artery. A branch of this artery enters the prostate laterally at the prostatovesical junction. The prostatic venous plexus receives the deep dorsal penile vein and the veins of the base of the bladder. The vesicle and internal iliac veins receive most of the venous blood.

According to Walsh, NVB is located between 2 layers of the lateral pelvic fascia (levator fascia-lateral and prostatic fascia-medial). He states that during a nerve sparing procedure, the prostatic fascia must remain on the prostate. ${ }^{[12]}$ Intrafascial dissection should be done between the prostatic capsule and periprostatic fascia, with interfacial dissection between periprostatic fascia and levator muscle fascia. We described all fascial layers, which were critical points for nerve sparing surgery.

\section{Conclusion}

The identification of anatomical landmarks in nervesparing radical prostatectomy in cadaver is an effective method to increase anatomical knowledge. The bloodless area of cadaver provides good visual aspect to determine exact localizations of anatomic landmarks. We suggest urologists to work collaboratively with anatomists and to act nSRP in cadaver before the live surgery.

\section{References}

1. Stolzenburg JU, Schwalenberg T, Horn LC, et al. Anatomicat landmarks of radical prostatectomy. Eur Urol 2007,51:629-39.

2. Stolzenburg JU, Rabenlat R, Do M, et al. Intrafascial nevre-sparing endoscopic extraperitoneal radical prostatectomy. Eur Urol 2008;53:931-40.

3. Catalona WJ, Carvalhal GF, Mager DE, et al. Potency, continence and complication rates in 1870 consecutive radical retropubic prostatectomy. J Urol 1999;162:433-8.

4. Rabbani F, Stapleton AM, Kattan MW, et al. Factors predicting recovery of erections after radical prostatectomy. J Urol 2000;164:1929-34.

5. D'Amico AV, WhittingtonR, Malkowicz SB, et al. Biochemical outcome after radical prostatectomy or external beam radiation therapy for patients with clinically localized prostate carcinoma in the prostate specific antigen era. Cancer 2002;95:281-6.

6. Hull GW, Rabbani F, Abbas F, et al. Cancer control with radical prostatectomy alone in 1000 consecutive patients. J Urol 2002;167:528-34

7. Roehl KA, Han M, Ramos CG, et al. Cancer progression and survival rates following anatomical radical retropubic prostatectomy in 3,478 consecutive patients: long-term results. J Urol 2004;172:910-4.

8. Michl UH, Friedrich MG, Graefen M, et al. Prediction of postoperative sexual function after nevre sparing radical retropubic prostatectomy. J Urol 2006;176:227-31.

9. Federatice Committee of Anatomical Terminology (FCAT). Terminologia anatomica. New York: Thieme; 1998.

10. Walz J, Graefen M, Huland H. Basic principals of anatomy for optimal surgical treatment of prostate cancer. World J Urol 2007;25:31-8.

11. McCarthy JF, Catalona WJ. Nerve sparing radical prostatectomy. In: Marshal FF, ed. Textbook of Operative Urology. Philadelphia: WB Saunders; 1996. p. 537-44.

12. Walsh PC. Anatomical radical prostatectomy: evolution of the surgical technique. J Urol 1998;169:2418-24.

13. Menon M, Tewari A, Peabody JO, et al. Vattikuti Institute prostatectomy: technique. J Urol 2003;169:2289-92.

14. Costello AJ, Brooks M, Cole OJ. Anatomical studies of the neurovascular bundle and the cavernosal nerves. BJU Int 2004;94:1071-6.

15. Kiyoshima K, Yokomizo A, Yoshida T, et al. Anatomical features of periprostatic tissue and its surroundings: a histological analysis of 79 radical retropubic prostatectomy specimens. Jpn Clin Oncol 2004;34:463-8.

16. Matusz P. Misleading Latin/English equivalents for some liver terms in Terminologia Anatomica. Anat Sci Educ 2010;3:156-7.

17. Myers RP, Cheville JG, Pawlina W. Making anatomic terminology of the prostate and contiguous structures clinically useful: historical review and suggestions for revision in the 21st century. Clin Anat 2010; 23:18-29.

18. Takenaka A, Hara R, Soga H, et al. A novel technique for approaching the endopelvic fascia in retropubic radical prostatectomy, based on an anatomical study of fixed and fresh cadavers. BJUIInt 2005;95:766-71.

19 Steiner MS. Continence-preserving anatomic radical retropubic prostatectomy. Urology 2000;5 5:427-35.

20. Myers RP. Practical surgical anatomy for radical prostatectomy. Urol Clin North Am 2001;28:473-90.

21. Walsh PC. Anatomic radical retropubic prostatectomy. In: Walsh PC, Retik AB, Vaugh ED Jr., Wein AJ, editors. Campbell's Urology. 8th ed. Vol. 4. Philadelphia: Saunders; 2002. p. 3107-29.

22. Myers RP, Villers A. Prostate Cancer: Principles and Practice. London: Taylor\&Francis; 2006.

23. Poore RE, McCullough DL, Jarow JP. Puboprostatic ligament sparing improves urinary continence after radical retropubic prostatectomy. Urology 1998;51:67-72.

24. Myers RP. Detrusor apron, associated vascular plexus, and avascular plane: relevance to radical retropubic prostatectomy - anatomic and surgical commentary. Urology 2002;59:472-9.

25. Song LJ, Lu HK, Wang JP, et al. Cadaveric study of nerves supplying the membranous urethra. Neurourol Urodynam 2010;29:592-5.

26. Graefen M, Walz J, Huland H. Open retropubic nerve sparing radical prostatectomy. Eur Urol 2006;49:38-48.

27. Mauroy B, Demondion X, Drizenko A, et al. The inferior hypogastric plexus (pelvic plexus): its importance in neural preservation techniques. Surg Radiol Anat 2003;25:6-15.
Correspondence to: Ilkan Tatar, MD, Assoc. Prof. Department of Anatomy, Faculty of Medicine, Hacettepe University, 06100 Sinhiye, Ankara

Phone: +90 3123052114 10; Fax: +90 3123107169

e-mail: ilkan@hacettepe.edu.tr

Conflict of interest statement: No conflicts declared. 